\title{
Characterisation of classical enterotoxins, virulence activity, and antibiotic susceptibility of Staphylococcus aureus isolated from Thai fermented pork sausages, clinical samples, and healthy carriers in northeastern Thailand
}

\author{
Wanwisa Sankomkai ${ }^{1}$, Wongwarut Boonyanugomol ${ }^{1,2 \bowtie}$, Kairin Kraisriwattana ${ }^{1}$, \\ Julalak Nutchanon $^{1}$, Kraisorn Boonsam ${ }^{3}$, Sasalux Kaewbutra ${ }^{1,2}$, Warawan Wongboot ${ }^{4}$ \\ ${ }^{1}$ Unit of Water and Food Analysis, Division of Research, ${ }^{2}$ Department of Sciences and Liberal Arts, \\ Mahidol University, Amnatcharoen Campus, Amnatcharoen, 37000, Thailand \\ ${ }^{3}$ Microbiology Laboratory, Division of Clinical Pathology, Amnatcharoen Hospital, Amnatcharoen, 37000, Thailand \\ ${ }^{4}$ National Institute of Health, Department of Medical Sciences, Ministry of Public Health, Nonthaburi, 11000, Thailand \\ Wongwarut.boo@mahidol.edu
}

Received: October 25, 2019 Accepted: May 13, 2020

\begin{abstract}
Introduction: Contamination by Staphylococcus aureus of food produced from animal sources may have diverse and multifactorial causes that depend on geographical distribution. The goal of this study was to isolate and characterise $S$. aureus strains from contaminated fermented pork sausage, which is a local food of northeastern Thailand. Material and Methods: S. aureus strains were isolated from local pork sausage, and the presence of classical enterotoxins was determined by PCR and reversed passive latex agglutination. These results were compared with strains derived from hospitalised patients and healthy carriers. Additionally, production of extracellular enzymes and haemolysin, biofilm formation, and antibiotic susceptibility were assessed. Results: S. aureus was identified in 36 sausage isolates (60\%). The strains positive for staphylococcal enterotoxin A were more frequently found in isolates from sausage and healthy carriers than in those from patients. All tested $S$. aureus strains were positive for DNase, lipase, proteinase, haemolysin, and biofilm formation; notably, strains isolated from food and healthy carriers displayed similar values. Most isolates were resistant to penicillin and ampicillin, while none were to methicillin. Conclusions: Thai fermented pork sausages are associated with a high risk of staphylococcal food poisoning, which may be linked to contamination caused by carriers. Dissemination of knowledge regarding best practices in sanitation and hygiene is important in local communities.
\end{abstract}

Keywords: food, pork, Staphylococcus aureus enterotoxins, Thailand.

\section{Introduction}

Staphylococcus aureus is a facultative anaerobic Gram-positive bacterium and a well-known causative agent of community or hospital-acquired infections and food poisoning. This bacterium is found widely in humans and animals (mainly colonising the nares and skin), the environment, and food produced from raw materials of animal origin, such as pork, beef, or chicken (18). Its prevalence contributes to worldwide foodborne diseases resulting from food contamination via poor slaughtering sanitation or inappropriate food processing, including food handling, mincing, preparation, wrapping, and storage (11). Fermented pork sausage, naem moo, is a local food of northeastern Thailand. It has a sour flavour that the local population prefers for raw consumption. Raw minced pork is commonly used as the main ingredient and is mixed with several local spices. It has been suggested that this local food could be contaminated with several pathogens, including $S$. aureus, as a result of unclean ingredients or inappropriate processing. Characterisation 
of $S$. aureus contaminants of this local food may lead to the development of strategies for surveillance and prevention of food poisoning.

Some enterotoxigenic $S$. aureus strains associated with food poisoning have been shown to produce staphylococcal enterotoxins (SEs), members of the large family of superantigenic toxins (2). SEs are highly heat stable, are not degraded by cooking, and also persist through harsh environmental conditions such as freezing and drying (16). More than 20 $S$. aureus enterotoxins have been investigated, of which the classical SEs are considered to be these five: SEA, SEB, SEC, SED, and SEE $(14,16)$. They are the most common causes of staphylococcal food poisoning, and among them, SEA is particularly frequent (24). SEs have been documented to induce several clinical manifestations following a short incubation period, including fever, nausea, vomiting, and diarrhoea. Some $S$. aureus strains produce an enterotoxin-like protein such as toxic shock syndrome toxin-1 (TSST-1, SEF), a manifestation featuring severe, acute symptoms and causing the dysfunction of multi-organ systems (7). In addition to enterotoxins, $S$. aureus strains produce haemolysin (pore-forming toxin) and several extracellular enzymes (including coagulase, protease, lipase, and DNase), and can form biofilms; these features are thought to facilitate survival and growth under a variety of stressful conditions and have associated consequences, such as immune evasion, bacterial dissemination, food spoilage, and tolerance to cold temperatures or high concentrations of salt and sugar during food production $(8,15)$.

To date, no studies have been performed to characterise $S$. aureus contaminants of Thai fermented pork sausage, which is popularly consumed raw in northeastern Thailand. This study aimed to isolate $S$. aureus from Thai fermented pork sausage and subsequently determine the presence of classical SE genes, comparing their prevalence against clinical and healthy carrier strains. Production of haemolysin and extracellular enzymes, biofilm formation, and antibiotic susceptibility of isolates obtained from three different sources were also investigated. Such determination of the genetic and phenotypic characteristics of $S$. aureus isolates is important for epidemiological surveillance and prevention of food contamination, especially in local foods produced from raw materials of animal origin. In addition, knowledge concerning antibiotic resistance in $S$. aureus strains from food samples may be beneficial for the control of antibiotic usage in local communities or on livestock farms.

\section{Material and Methods}

S. aureus isolation from food samples. A total of 60 Thai fermented pork sausage samples were collected from local retail markets in the Amnatcharoen municipal area, which is located in northeastern Thailand. For $S$. aureus isolation, $10 \mathrm{~g}$ of Thai fermented pork sausage was transferred to $90 \mathrm{~mL}$ of tryptic soy broth (TSB) (Himedia, Mumbai, India) containing $10 \% \mathrm{NaCl}$ and blended for $10 \mathrm{~min}$ by laboratory flapping homogenizer (HG400VW, Wiggens, Straubenhardt, Germany). Ten millilitres of suspension were inoculated into $90 \mathrm{~mL}$ of TSB (containing $10 \% \mathrm{NaCl}$ ), and the solution was incubated at $37^{\circ} \mathrm{C}$ for $24 \mathrm{~h}$. Then, one loopful of enrichment broth was streaked on Baird-Parker agar (BPA) (Himedia) containing egg yolk tellurite emulsion (FD046, Himedia); this was incubated at $37^{\circ} \mathrm{C}$ for $48 \mathrm{~h}$. Typical black shiny colonies with an opaque zone were selected and subsequently subjected to Gram staining and biochemical determination with coagulase, phenol red (PR) glucose, and PR mannitol tests.

The anterior nares of 30 healthy volunteers were swabbed for $S$. aureus isolation. The swabs were streaked on BPA (supplemented with egg yolk tellurite emulsion) and incubated at $37^{\circ} \mathrm{C}$ for $48 \mathrm{~h}$. Typical colonies were identified by Gram staining and biochemical testing.

Clinical strains of $S$. aureus isolated from 54 hospitalised patients were provided by the Clinical Microbiology Laboratory at Amnatcharoen Hospital, Amnatcharoen, Thailand. All S. aureus isolates were previously confirmed by biochemical testing.

Detection of classical SE genes (sea-see) and toxic shock syndrome toxin-1 (tsst-1). Genomic DNA of $S$. aureus was extracted using a Genomic DNA Extraction Kit (Bio-Helix, Keelung, Taiwan) according to the manufacturer's instructions. DNA pellets were dissolved in TE buffer and stored at $-20^{\circ} \mathrm{C}$ until use. The presence of a gene encoding thermostable nuclease (nuc) was confirmed in all isolates by a PCR assay. Classical enterotoxin-associated genes (sea, seb, sec, sed, and see) and tsst-1 (sef) were detected by a PCR assay using primers with sequences shown in Table 1 and under conditions as given in previously published reports $(10,12,20,28)$. PCR solutions consisted of One PCR Plus reaction mixture (GeneDireX, Keelung, Taiwan) and $0.5 \mu \mathrm{M}$ of each primer. Amplification was performed in a T100 Thermal Cycler (Bio Rad Laboratories, Hercules, CA, USA). The resulting PCR products were evaluated by $1.5 \%$ agarose gel electrophoresis and visualised with a UV illuminator. The reference S. aureus strains DMST 19376 (sea), DMST 19377 (seb), DMST 19378 (sec), and DMST 19379 (sed) were provided by the Department of Medical Sciences Thailand Culture Collection and were used as positive controls (sea-sed). In addition, the in-house S. aureus clinical strains SP1709 and P950 were used as positive controls for see and tsst-1, respectively.

The toxin production of all $S$. aureus isolates positive for classical enterotoxin genes was determined using reversed passive latex agglutination (RPLA) against staphylococcal enterotoxins A, B, C, and D, according to the manufacturer's instructions (Oxoid, Wesel, Germany). 
Table 1. Primer sequences used for detection of classical SE genes (sea-see) and the gene encoding toxic shock syndrome tsst-1

\begin{tabular}{|c|c|c|c|}
\hline Gene & Primer sequences & Product size (bp) & Reference \\
\hline \multirow[t]{2}{*}{ nuc } & GCGATTGATGGTGATACGGTT & 279 & $(12)$ \\
\hline & AGCCAAGCCTTGACGAACTAAAGC & & \\
\hline \multirow[t]{2}{*}{ sea } & ACCGTTTCCAAAGGTACTGTA & 135 & $(28)$ \\
\hline & TGGTACACCAAACAAAACAGC & & \\
\hline \multirow[t]{2}{*}{$s e b$} & CCTAAACCAGATGAGTTGCAC & 592 & $(28)$ \\
\hline & CAGGCATCATGTCATACCAAA & & \\
\hline \multirow[t]{2}{*}{$\sec$} & AGATGAAGTAGTTGATGTGTATGG & 454 & $(20)$ \\
\hline & CTTCACACTTTTAGAATCAACCG & & \\
\hline \multirow[t]{2}{*}{ sed } & GCTTGTACATATGGAGGTGTCA & 263 & $(28)$ \\
\hline & GACCCATCAGAAGAATCAAACT & & \\
\hline \multirow[t]{2}{*}{ see } & CAGTACCTATAGATAAAGTTAAAACAAGC & 178 & $(10)$ \\
\hline & TAACTTACCGTGGACCCTTC & & \\
\hline \multirow[t]{2}{*}{ tsst-1 } & GGCAGCATCAGCCTTATAATTT & 371 & $(28)$ \\
\hline & GTGGATCCGTCATTCATTGTT & & \\
\hline
\end{tabular}

Determination of extracellular enzyme and haemolysin production in $S$. aureus isolates. $S$. aureus isolates were cultured in TSB at $37^{\circ} \mathrm{C}$ for $24 \mathrm{~h}$. Subsequently, the bacterial suspensions were diluted to $0.5 \mathrm{McF}$ arland $\left(\sim 1 \times 10^{8}\right.$ cells $\left./ \mathrm{mL}\right)$ and used to determine the production of extracellular enzymes and haemolysin by dropping $10 \mu \mathrm{L}$ of bacterial suspension onto skim milk agar (for protease production), tributyrin agar (for lipase production), DNase agar (for DNase production), or blood agar base containing 5\% human blood (for haemolysin production) (all from Himedia). All culture plates were incubated at $37^{\circ} \mathrm{C}$ for $48 \mathrm{~h}$. The production of extracellular enzymes and haemolysin was evaluated by measuring clear zone diameters in $\mathrm{mm}$ in triplicate independent experiments. To determine the production of DNase, $15 \mathrm{~mL}$ of $1 \mathrm{~N} \mathrm{HCl}$ was poured onto agar plates, and excess $\mathrm{HCl}$ was aspirated; clear zones around colonies indicated DNase activity.

Determination of biofilm formation by $S$. aureus isolates. $S$. aureus isolates were inoculated into LuriaBertani broth (LB) (Himedia) and incubated at $37^{\circ} \mathrm{C}$ for $24 \mathrm{~h}$ in a shaking incubator (180 rpm). Each inoculum was adjusted to a bacterial turbidity of 0.5 McFarland $\left(\sim 1 \times 10^{8} \mathrm{CFU} / \mathrm{mL}\right)$. To evaluate biofilm formation, $200 \mu \mathrm{L}$ was added into each well of a polystyrene 96-well microtiter plate and incubated at $37^{\circ} \mathrm{C}$ for $48 \mathrm{~h}$, as described in a previous protocol, with slight modification (30). After the incubation, nonattached bacteria were removed from the wells, and the plates were washed three times with PBS. Afterwards, $200 \mu \mathrm{L}$ of $0.1 \%$ crystal violet solution was added into each well, and the plate was incubated at room temperature for $30 \mathrm{~min}$. Next, the suspension of crystal violet was removed, and all wells were washed three times with PBS for $5 \mathrm{~min}$ with shaking. The stained samples were air dried for $1 \mathrm{~h}$ and then solubilised by incubation with $200 \mu \mathrm{L}$ of absolute ethanol for $10 \mathrm{~min}$. The absorbance of solubilised crystal violet was measured at $570 \mathrm{~nm}$ using a microplate reader (SPECTROstar NANO, BMG Labtech, Ortenberg, Germany). Sterile LB was used as a blank negative control. Biofilm production was quantified based on triplicate independent experiments. The criteria used for categorising isolates in terms of biofilm production were those in the previous report of Singh et al. (25). $\mathrm{OD}_{\text {cutoff }}=$ average OD of negative control $+3 \times$ standard deviation (SD) of ODs of negative control;

$<\mathrm{OD}_{\text {cutoff }}=$ biofilm non-former;

$\geq \mathrm{OD}_{\text {cutoff }}$ and $<2 \times \mathrm{OD}_{\text {cutoff }}=$ weak biofilm former;

$\geq 2 \times \mathrm{OD}_{\text {cutoff }}$ and $<4 \times \mathrm{OD}_{\text {cutoff }}=$ moderate biofilm former;

$\geq 4 \times \mathrm{OD}_{\text {cutoff }}=$ strong biofilm former.

Antibiotic susceptibility testing. All strains of S. aureus (0.5 McFarland) were tested for antibiotic susceptibility using the Kirby-Bauer protocol, which was conducted on Mueller-Hinton agar (MHA) (Himedia). A panel of 13 antibiotics was used to test drug susceptibility based on the Clinical and Laboratory Standard Institute guidelines (4). This panel (Oxoid, Basingstoke, UK) included: (1) $\beta$-lactams, consisting of penicillin (10 $\mathrm{U})$, ampicillin $(10 \mu \mathrm{g})$, amoxicillin/clavulanic acid $(30 \mu \mathrm{g})$, ceftriaxone $(30 \mu \mathrm{g})$, cephazolin $(30 \mu \mathrm{g})$, ceftazidime $(30 \mu \mathrm{g})$, and cefoxitin $(30 \mu \mathrm{g})$; (2) lincosamides, consisting of clindamycin $(10 \mu \mathrm{g})$; (3) macrolides, consisting of erythromycin $(30 \mu \mathrm{g})$; (4) aminoglycosides, consisting of gentamicin $(10 \mu \mathrm{g})$; (5) phenicols, consisting of chloramphenicol $(30 \mu \mathrm{g})$; (6) glycopeptides, consisting of vancomycin $(30 \mu \mathrm{g})$; and (7) sulphonamides, consisting of trimethoprim/sulfamethoxazole $(25 \mu \mathrm{g})$. Notably, cefoxitin has been suggested for the detection of methicillin resistance in S. aureus (4).

Data analysis. The chi-squared test was used to analyse differences in the prevalence of SEs among the three experimental groups. Differences in clear zones and biofilm formation among the various groups were determined by Student's $t$-test. P values less than 0.05 were considered to be statistically significant.

\section{Results}

Isolation of $S$. aureus from Thai fermented pork sausages. $S$. aureus strains were detected in 36 (60\%) samples out of the 60 samples tested. Among the 
30 healthy volunteers tested, this study found $S$. aureus in $10(33.33 \%)$. PR glucose, PR mannitol, and coagulase tests were positive for all $S$. aureus isolates. Additionally, positive results with $S$. aureus-specific primers (nuc) were obtained (Fig. 1).

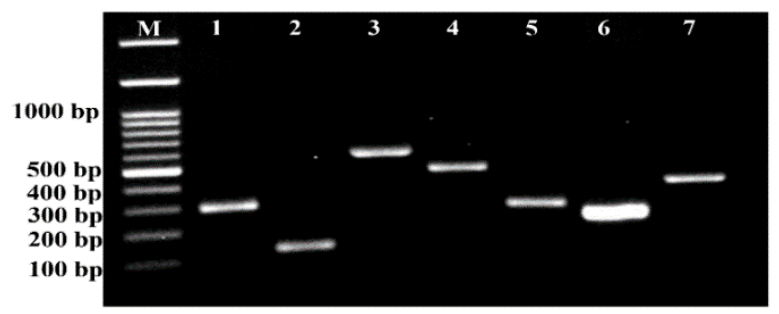

Fig. 1. PCR amplification of a $S$. aureus specific gene (nuc), classical SE genes (sea-see), and the tsst-1 gene. Lane M - 100 bp DNA marker; Lanes 1-7 - nuc, sea, seb, sec, sed, see, and tsst-1, respectively

Prevalence of classical SE (sea-see) and toxic shock syndrome toxin-1 (tsst-1) genes. PCR assays (Fig. 1) were used to determine the presence of classical SE (sea, seb, sec, sed, and see) and tsst-1 genes in the $36 \mathrm{~S}$. aureus isolates derived from Thai fermented pork sausages. This was compared to their prevalence in strains isolated from clinical specimens $(\mathrm{n}=54)$ and healthy carriers $(\mathrm{n}=10)$, as summarised in Table 2. The SE genes were detected in $47 \%$ (sea), $11 \%$ (seb), 0\% (sec), 0\% (sed), and 3\% (see) of Thai fermented pork sausage samples. Additionally, two or three SE genes co-occurred in several samples: sea and seb (5\%), seb and sec (17\%), and sea, seb and sec (11\%). However, tsst- 1 was not detected either alone or in combination with other genes. Among hospitalised patients, SE genes were respectively detected in $13 \%$ (sea), 33\% (seb), 2\% (sec), 0\% (sed), and 2\% (see) of $S$. aureus isolates. These strains also exhibited genes in combination, including sea and seb (2\%), seb and sec (17\%), sea, seb and sec (4\%), and seb, sec and tsst-1 $(7 \%)$. Ten isolates from healthy carriers were positive for sea (40\%), seb (10\%), sea and seb (10\%), and sea, seb and $\sec (10 \%)$.

Production of classical enterotoxins (SEA-SED) by PCR-positive isolates was confirmed by RPLA testing. SEA-positive strains were highly frequent in isolates from Thai fermented pork sausages (61\%) and healthy carriers $(60 \%)$, and had low frequency in isolates from hospitalised patients $(7 \%)(\mathrm{P}<0.05)$.

Production of extracellular enzymes and haemolysin by $S$. aureus isolates. All $S$. aureus isolates from the three different groups were positive for DNase, lipase, protease, and haemolysin production. For strains from Thai fermented pork sausages, the average clear zones for DNase, lipase, protease, and haemolysin were 20.6, 18.2, 13, and $12.5 \mathrm{~mm}$, respectively, and were similar to the average clear zones for strains from healthy carriers which were $21.6 \mathrm{~mm}, 18 \mathrm{~mm}, 13.9 \mathrm{~mm}$, and $12.9 \mathrm{~mm}$ (Fig. 2). Additionally, significantly higher production was observed for strains from hospitalised patients (DNase $29.8 \mathrm{~mm}$, lipase $20.4 \mathrm{~mm}$, protease $15.3 \mathrm{~mm}$, haemolysin $15.1 \mathrm{~mm}$ ) relative to other tested strains $(\mathrm{P}<0.05)$.

Biofilm formation by $\boldsymbol{S}$. aureus isolates. We also determined the biofilm formation capability of isolated $S$. aureus strains and categorised them accordingly as biofilm non-formers, weak biofilm formers, moderate biofilm formers, and strong biofilm formers (Table 3 ). Most isolates were determined to be strong biofilm formers, but two strains detected in Thai fermented pork sausages $(6 \%)$ were classified as moderate biofilm formers.

Although most strains were strong biofilm formers, we found that the production of biofilm in $S$. aureus isolated from hospitalised patients (mean OD $2.73 \pm 0.37$ ) was significantly higher than that of strains isolated from Thai fermented pork sausages (mean OD $1.62 \pm 0.36$ ) or healthy carriers (mean OD $1.72 \pm 0.32)$, as shown in Fig. 3 .

Table 2. Detection of classical SE and tsst-1 genes by PCR and of classical SE production by RPLA

\begin{tabular}{|c|c|c|c|c|c|c|}
\hline \multirow{2}{*}{$\begin{array}{l}\text { S. aureus enterotoxin } \\
\text { genotype }\end{array}$} & \multicolumn{2}{|c|}{ Thai fermented pork sausages $(n=36)$} & \multicolumn{2}{|c|}{ Hospitalised patients $(n=54)$} & \multicolumn{2}{|c|}{ Healthy carriers $(n=10)$} \\
\hline & PCR & RPLA & PCR & RPLA & PCR & RPLA \\
\hline sea & $17(47 \%)$ & $22(61 \%)^{\mathrm{a}}$ & $7(13 \%)$ & $4(7 \%)$ & $4(40 \%)$ & $6(60 \%)^{b}$ \\
\hline$s e b$ & $4(11 \%)$ & $15(42 \%)$ & $18(33 \%)$ & $15(28 \%)$ & $1(10 \%)$ & $3(30 \%)$ \\
\hline sec & $0(0 \%)$ & $10(28 \%)$ & $1(2 \%)$ & $6(11 \%)$ & $0(0 \%)$ & $1(10 \%)$ \\
\hline sed & $0(0 \%)$ & $0(0 \%)$ & $0(0 \%)$ & $0(0 \%)$ & $0(0 \%)$ & $0(0 \%)$ \\
\hline see & $1(3 \%)$ & ND & $1(2 \%)$ & ND & $0(0 \%)$ & ND \\
\hline tsst-1 & $0(0 \%)$ & - & $0(0 \%)$ & - & $0(0 \%)$ & - \\
\hline sealseb & $2(5 \%)$ & - & $1(2 \%)$ & - & $1(10 \%)$ & - \\
\hline $\mathrm{seb} / \mathrm{sec}$ & $6(17 \%)$ & - & $9(17 \%)$ & - & $0(0 \%)$ & - \\
\hline sea/seb/sec & $4(11 \%)$ & - & $2(4 \%)$ & - & $1(10 \%)$ & - \\
\hline seb/sec/tsst-1 & $0(0 \%)$ & - & $4(7 \%)$ & - & $0(0 \%)$ & - \\
\hline Total & $34(94 \%)$ & & $43(80 \%)$ & & $7(70 \%)$ & \\
\hline
\end{tabular}


Determination of antibiotic susceptibility in S. aureus from different sources. Susceptibility was examined for seven groups of antibiotics (Table 4). The 36 strains of $S$. aureus isolated from Thai fermented pork sausage samples mainly exhibited resistance against the $\beta$-lactam group. Specifically, resistance to penicillin, ampicillin, and amoxicillin/clavulanic acid was detected in $83 \%, 72 \%$, and $8 \%$ of those isolates respectively, but all were susceptible to ceftriaxone, cephazolin, ceftazidime, and cefoxitin. All isolates from sausage were also found to be susceptible to other antibiotic groups, including lincosamides (clindamycin), macrolides (erythromycin), aminoglycosides (gentamicin), phenicols (chloramphenicol), glycopeptides (vancomycin), and sulphonamides (trimethoprim/sulfamethoxazole).

Similar resistance patterns were found in $S$. aureus isolates from hospitalised patients. These isolates were resistant to the $\beta$-lactam group, with $87 \%, 87 \%$, and $2 \%$ of isolates being resistant to penicillin, ampicillin, and amoxicillin/clavulanic acid, respectively, and susceptible to ceftriaxone, cephazolin, ceftazidime, and cefoxitin. Fewer than $10 \%$ of patient isolates were resistant to the lincosamides (clindamycin), macrolides (erythromycin), or phenicols (chloramphenicol) groups. All patient isolates were susceptible to the aminoglycosides (gentamicin), glycopeptides (vancomycin), and sulphonamides (trimethoprim/ sulfamethoxazole).

$S$. aureus strains isolated from healthy carriers were also resistant to the $\beta$-lactam group, including penicillin (80\%), ampicillin $(70 \%)$, and amoxicillin/ clavulanic acid (10\%), but were susceptible to ceftriaxone, cephazolin, ceftazidime, and cefoxitin. Ten percent of these isolates exhibited resistance to the lincosamide and macrolide groups, including clindamycin and erythromycin. All isolates were also found to be susceptible to the aminoglycosides (gentamicin), phenicols (chloramphenicol), glycopeptides (vancomycin), and sulphonamides (trimethoprim/sulfamethoxazole).

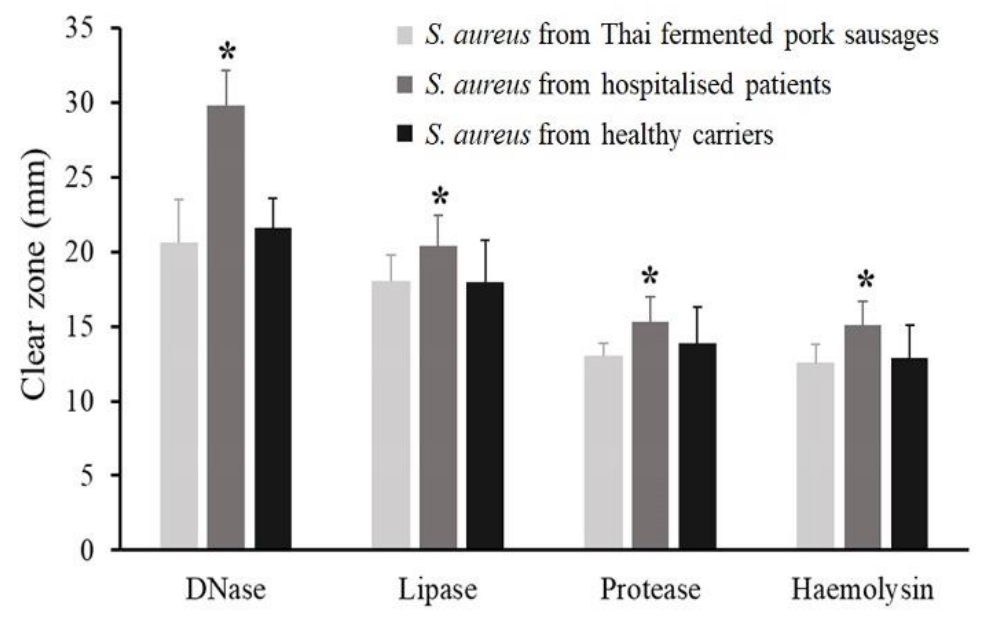

Fig. 2. Production of extracellular enzymes (DNase, lipase, protease) and haemolysin by $S$. aureus strains isolated from three different sources. Data are presented as the mean diameter of clear zones surrounding colonies $(\mathrm{mm})$, determined from triplicate independent experiments $* \mathrm{P}<0.05$ is considered to be statistically significant in between-group comparisons

Table 3. Grading of biofilm formation in S. aureus strains isolated from three different sources

\begin{tabular}{lllll}
\hline & & & \multicolumn{2}{l}{ S. aureus isolates } \\
\cline { 3 - 5 } OD ranges $(570 \mathrm{~nm})$ & Biofilm quantity grade & $\begin{array}{l}\text { Thai fermented pork } \\
\text { sausages }(\mathrm{n}=36)\end{array}$ & $\begin{array}{l}\text { Hospitalised patients } \\
(\mathrm{n}=54)\end{array}$ & $\begin{array}{l}\text { Healthy carriers } \\
(\mathrm{n}=10)\end{array}$ \\
\hline$<0.19$ & Biofilm non-former & $0(0 \%)$ & $0(0 \%)$ & $0(0 \%)$ \\
\hline$\geq 0.19$ and $<0.38$ & Weak biofilm former & $0(0 \%)$ & $0(0 \%)$ & $0(0 \%)$ \\
\hline$\geq 0.38$ and $<0.76$ & Moderate biofilm former & $2(6 \%)$ & $0(0 \%)$ & $0(0 \%)$ \\
\hline$\geq 0.76$ & Strong biofilm former & $34(94 \%)$ & $54(100 \%)$ & $10(100 \%)$ \\
\hline
\end{tabular}

The OD cutoff was determined using the average OD of negative control $+(3 \times$ standard deviation (SD) of ODs of negative control) 


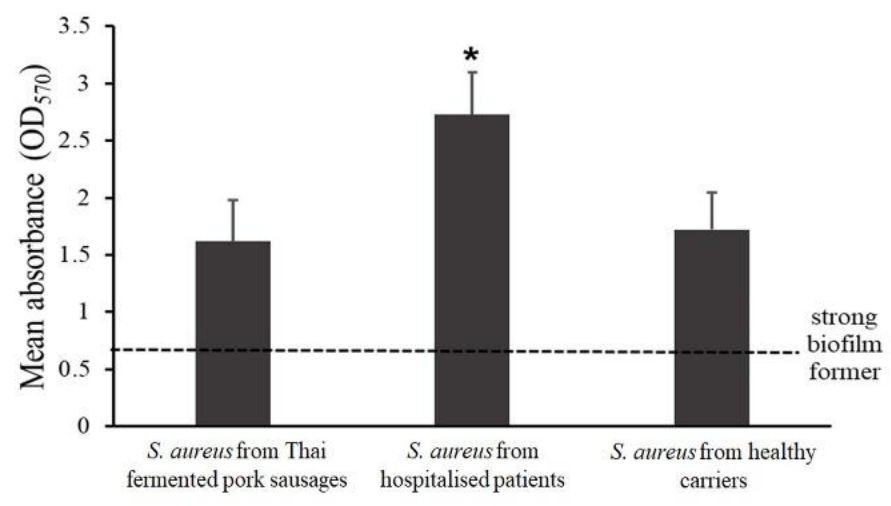

Fig. 3. Biofilm formation was evaluated by crystal violet staining in triplicate independent experiments. Absorbance at $570 \mathrm{~nm}$ was measured with a microplate reader. Isolates with $\mathrm{OD}_{570}$ values $\geq 0.76$ were considered to be strong biofilm formers. $* \mathrm{P}<0.05$ was considered to be significantly different in between-group comparisons

Table 4. Antibiotic resistance in $S$. aureus strains isolated from samples of Thai fermented pork sausage, hospitalised patients, and healthy carriers

\begin{tabular}{|c|c|c|c|c|}
\hline \multirow[b]{2}{*}{ Antibiotic group } & \multirow[b]{2}{*}{ Antibiotic } & \multicolumn{3}{|c|}{ Antibiotic resistance in $S$. aureus isolates } \\
\hline & & $\begin{array}{l}\text { Thai fermented } \\
\text { pork sausages } \\
(\mathrm{n}=36)\end{array}$ & $\begin{array}{l}\text { Hospitalised } \\
\text { patients } \\
(\mathrm{n}=54)\end{array}$ & $\begin{array}{l}\text { Healthy } \\
\text { carriers } \\
(\mathrm{n}=10)\end{array}$ \\
\hline \multirow[t]{7}{*}{$\beta$-lactams } & penicillin & $30(83 \%)$ & $47(87 \%)$ & $8(80 \%)$ \\
\hline & ampicillin & $26(72 \%)$ & $47(87 \%)$ & $7(70 \%)$ \\
\hline & amoxicillin/clavulanic acid & $3(8 \%)$ & $1(2 \%)$ & $1(10 \%)$ \\
\hline & ceftriaxone & $0(0 \%)$ & $0(0 \%)$ & $0(0 \%)$ \\
\hline & cephazolin & $0(0 \%)$ & $0(0 \%)$ & $0(0 \%)$ \\
\hline & ceftazidime & $0(0 \%)$ & $0(0 \%)$ & $0(0 \%)$ \\
\hline & cefoxitin & $0(0 \%)$ & $0(0 \%)$ & $0(0 \%)$ \\
\hline Lincosamides & clindamycin & $0(0 \%)$ & $4(7 \%)$ & $1(10 \%)$ \\
\hline Macrolides & erythromycin & $0(0 \%)$ & $4(7 \%)$ & $1(10 \%)$ \\
\hline Aminoglycosides & gentamicin & $0(0 \%)$ & $0(0 \%)$ & $0(0 \%)$ \\
\hline Phenicols & chloramphenicol & $0(0 \%)$ & $2(4 \%)$ & $0(0 \%)$ \\
\hline Glycopeptides & vancomycin & $0(0 \%)$ & $0(0 \%)$ & $0(0 \%)$ \\
\hline Sulphonamides & trimethoprim/sulfamethoxazole & $0(0 \%)$ & $0(0 \%)$ & $0(0 \%)$ \\
\hline
\end{tabular}

\section{Discussion}

Due to the ability of $S$. aureus to colonise the nares and skin of animals, several foods produced from raw materials originating from animals such as poultry, cattle, and swine have been assumed to be reservoirs for $S$. aureus contamination. There are many risk factors associated with food produced from pathogencontaminated animals, such as poor sanitary conditions in slaughterhouses, unclean conditions in retail markets, and poor personal hygiene of food producers. In this study, we evaluated Thai fermented pork sausages sold in local retail markets of the Amnatcharoen municipal area, northeastern Thailand, and identified a high incidence of $S$. aureus contamination $(60 \%)$ that may cause food poisoning in consumers. The main ingredient of this local food consists of raw minced pork, and it is usually sold at open retail markets, creating a risk of contamination by various pathogens, including S. aureus. Furthermore, food producers usually touch raw minced pork with bare hands during food production, which increases the risk of $S$. aureus contamination.

Overall, classical SE genes were detected in $94 \%$ of strains from Thai fermented pork sausages, a frequency that is close to the prevalence in hospitalised patients $(80 \%)$ and in healthy carriers (70\%). However, detection of enterotoxin genes may not be indicative of enterotoxin production. Using RPLA assays, it was found that isolates from Thai fermented pork sausages and healthy carriers predominantly produced SEA, while clinical strains exhibited very low production of the same toxin. It is well-recognised that SEA-producing S. aureus strains are the most common toxin producers associated with food poisoning (17) and are responsible for food poisoning outbreaks worldwide, including in the USA (1). 
Different distributions of SE genes in contaminated foods have been reported worldwide. In Thailand, previous studies conducted in Khon Kaen province revealed a high prevalence of SEA-positive strains in food from different sources (Thai local food, seafood, fruit juice, and beverages) and in healthy carriers $(28,29)$. In China, the seb genotype was the most frequent enterotoxin gene in $S$. aureus isolated from pork products sampled from a slaughterhouse and terminal market (31). Meanwhile, in South Korea, $S$. aureus isolates derived from milk products produced two or more types of toxin (mainly SEA, SEB, and/or SEC), whereas isolates from raw meat and vegetables primarily produced SEA alone (22). We expect that Thai fermented pork sausages may become contaminated with SE-producing strains from carriers among slaughterhouse workers or food producers. However, Momoh et al. (21) reported that sea was the most common enterotoxin gene in $S$. aureus isolated from nasal swabs of pigs. Therefore, contamination by $S$. aureus flora that normally colonise pigs should also be considered. The varied prevalence of genes encoding SEs in strains colonising different foods, animals, humans, or geographical environments may be partially explained by the effect of mobile genetic elements (MGEs), which mediate horizontal gene transfer among staphylococci strains (19). The gene encoding tsst-1 or sef, which can cause serious morbidity and mortality, was found only in four isolates from hospitalised patients (where it occurred alongside seb and sec). The small number of tsst-1positive strains in this study may be due to this gene only being carried by a limited number of $S$. aureus strains overall (15). Furthermore, the marked predominance of tsst- 1 positivity in clinical strains indicates that hospitals may be an important reservoir of $S$. aureus-associated toxic shock syndrome. We did not determine the prevalence of new SEs in this study, both because their emetic activity is still not clear and because of limitations of the diagnostic RPLA kit.

Along with haemolysin, S. aureus can produce a large number of extracellular enzymes (such as DNase, lipase, and protease) which allow the bacteria to acquire nutrients necessary for survival, to propagate, and to evade the immune system (27). Preliminary screening of extracellular enzymes and haemolysin production was performed on the $S$. aureus isolates in this study. All $S$. aureus isolates secreted the investigated enzymes and haemolysin, but the clinical isolates derived from hospitalised patients collectively exhibited the highest levels of DNase, lipase, protease, and haemolysin production, while strains derived from food and healthy carriers showed equal expression levels. The significant production of extracellular enzymes and haemolysin by strains from hospitalised patients may indicate an adaptive response to stressful environments inside the human body (6).

Biofilm formation ability was also examined in all S. aureus isolates. Almost all were classified as strong biofilm producers, with only two strains being moderate producers. However, between-group comparisons showed significantly greater biofilm formation ability for $S$. aureus strains from hospitalised patients. The particularly strong biofilm formation of clinical strains may be a consequence of bacterial exposure to various immune factors and cytokines during infection. It is conceivable that exposure of $S$. aureus to inflammatory cytokines such as IL-1 $\beta$ and IFN- $\Upsilon$ promotes biofilm formation ability. Such exposure has been previously reported to induce significant stimulation of bacterial growth of planktonically- and biofilm-growing $S$. aureus strains both in vitro and in vivo $(5,9)$. However, the overall strong biofilm formation of isolates from food, patients, and general healthy carriers alike is an important concern; in accordance with previous work (23), we suggest that this capability may promote multidrug resistance and that awareness of this trait should be increased.

The antimicrobial susceptibility of all S. aureus isolates was also investigated in this study. We found a high rate of resistance against the $\beta$-lactam group antibiotics, especially penicillin and ampicillin. A few isolates were resistant to amoxicillin/clavulanic acid. Most $S$. aureus isolates were susceptible to lincosamides, macrolides, aminoglycosides, phenicols, glycopeptides, and sulphonamides. However, we identified six clinical isolates that exhibited multidrug resistance to $\beta$-lactams, lincosamides, macrolides, and phenicols. Fortunately, methicillin- or vancomycinresistant $S$. aureus (MRSA or VRSA) was not detected among the isolates in this study. Nevertheless, the existence of penicillin and ampicillin resistance in isolates derived from all three sources is of concern and may be due to the abundant usage of penicillin for production of swine, cattle, and poultry, especially in low- to middle- income countries. In Thailand, amoxicillin and ampicillin are widely sold in local grocery stores and general retail shops (13); furthermore, most antibiotics are easily available for home or farm use from retail pharmacies without a prescription (26). Thai adults were reported to have a low level of knowledge of antibiotics generally when this was tested by eliciting judgments of the truth or falsehood of prepared statements. This may indicate a large knowledge gap in the Thai population regarding the appropriate use of antibiotics (3). These previous observations cohere with the increasing antibiotic resistance in local Thai communities. Although MRSA strains were not found in this study, long term surveillance and frequent monitoring are necessary.

In summary, high levels of $S$. aureus contamination were detected in Thai fermented pork sausages, which are a local food of northeastern Thailand. We suggest that this local food may be a high-risk reservoir for staphylococcal food poisoning. SEA appeared to be the major SE in these sausages, similar to strains isolated from healthy carriers. This 
study may help us to better understand the distribution of the types of $S$. aureus in local foods produced from animals in northeastern Thailand, and may be useful as a basic database for further epidemiological surveillance and prevention of food poisoning. To reduce foodborne pathogen contamination in local foods, dissemination to local communities of knowledge of better sanitary and hygiene practices in slaughterhouses or food production operations, along with knowledge of antibiotic usage, should be accorded great importance.

Conflict of Interests Statement: The authors declare that there is no conflict of interests regarding the publication of this article.

Financial Disclosure Statement: This study was supported by a research grant from the Mahidol University, Amnatcharoen Campus, Thailand (AM14/2561).

Human Ethics Statement: This study was approved by the Human Ethics Committee of Mahidol University, Thailand, according to the Declaration of Helsinki and ICH GCP (COA no. MU-CIRB 2018/190.0111). Written informed consent was obtained from healthy volunteers prior to sample collection.

Animal Rights Statement: None required.

Acknowledgments: We acknowledge the Central Unit of Instrument and Equipment Services, Division of Research, Mahidol University, Amnatcharoen Campus, for their support of the instruments used throughout this study. We also thank the clinical microbiology staff for providing $S$. aureus clinical strains for this study.

\section{References}

1. Atanassova V., Meindl A., Ring C.: Prevalence of Staphylococcus aureus and staphylococcal enterotoxins in raw pork and uncooked smoked ham - a comparison of classical culturing detection and RFLP-PCR. Int J Food Microbiol 2001, 68, 105-113.

2. Balaban N., Rasooly A.: Staphylococcal enterotoxins. Int J Food Microbiol 2000, 61, 1-10.

3. Chanvatik S., Kosiyaporn H., Lekagul A., Kaewkhankhaeng W., Vongmongkol V., Thunyahan A., Tangcharoensathien V.: Knowledge and use of antibiotics in Thailand: A 2017 national household survey. PLoS One 2019, 14, e0220990.

4. Clinical and Laboratory Standards Institute: Performance standards for antimicrobial susceptibility testing; twenty-eighth informational supplement. Document M100-S27. CLSI, Wayne, 2018.

5. Di Domenico E.G., Cavallo I., Bordignon V., Prignano G., Sperduti I., Gurtner A., Trento E., Toma L., Pimpinelli F., Capitanio B., Ensoli F.: Inflammatory cytokines and biofilm production sustain Staphylococcus aureus outgrowth and persistence: a pivotal interplay in the pathogenesis of atopic dermatitis. Sci Rep 2018, 8, 9573.
6. Duong J., Booth S.C., McCartney N.K., Rabin H.R., Parkins M.D., Storey D.G.: Phenotypic and Genotypic Comparison of Epidemic and Non-Epidemic Strains of Pseudomonas aeruginosa from Individuals with Cystic Fibrosis. PLoS One 2015, 10, e0143466.

7. Fraser J., Arcus V., Kong P., Baker E., Proft T.: Superantigens powerful modifiers of the immune system. Mol Med Today 2000, 6, 125-132.

8. Galie S., Garcia-Gutierrez C., Miguelez E.M., Villar C.J., Lombo F.: Biofilms in the Food Industry: Health Aspects and Control Methods. Front Microbiol 2018, 9, 898.

9. Gutierrez Jauregui R., Fleige H., Bubke A., Rohde M., Weiss S., Forster R.: IL-1beta Promotes Staphylococcus aureus Biofilms on Implants in vivo. Front Immunol 2019, 10, 1082.

10. Johnson W.M., Tyler S.D., Ewan E.P., Ashton F.E., Pollard D.R., Rozee K.R.: Detection of genes for enterotoxins, exfoliative toxins, and toxic shock syndrome toxin 1 in Staphylococcus aureus by the polymerase chain reaction. J Clin Microbiol 1991, 29, 426-430.

11. Kadariya J., Smith T.C., Thapaliya D.: Staphylococcus aureus and staphylococcal food-borne disease: an ongoing challenge in public health. Biomed Res Int 2014, 2014, 827965.

12. Kateete D.P., Kimani C.N., Katabazi F.A., Okeng A., Okee M.S., Nanteza A., Joloba M.L., Najjuka F.C.: Identification of Staphylococcus aureus: DNase and Mannitol salt agar improve the efficiency of the tube coagulase test. Ann Clin Microbiol Antimicrob 2010, 9, 23.

13. Khamsarn S., Nampoonsak Y., Busamaro S., Tangkoskul T., Seenama C., Rattanaumpawan P., Boonyasiri A., Thamlikitkul V.: Epidemiology of Antibiotic Use and Antimicrobial Resistance in Selected Communities in Thailand. J Med Assoc Thai 2016, 99, 270-275.

14. Kim N.H., Yun A.R., Rhee M.S.: Prevalence and classification of toxigenic Staphylococcus aureus isolated from refrigerated ready-to-eat foods (sushi, kimbab and California rolls) in Korea. J Appl Microbiol 2011, 111, 1456-1464.

15. Kong C., Neoh H.M., Nathan S.: Targeting Staphylococcus aureus Toxins: A Potential form of Anti-Virulence Therapy. Toxins (Basel) 2016, 8, 72.

16. Le Loir Y., Baron F., Gautier M.: Staphylococcus aureus and food poisoning. Genet Mol Res 2003, 2, 63-76.

17. Ler S.G., Lee F.K., Gopalakrishnakone P.: Trends in detection of warfare agents. Detection methods for ricin, staphylococcal enterotoxin B and T-2 toxin. J Chromatogr A 2006, 1133, 1-12.

18. Lowder B.V., Guinane C.M., Ben Zakour N.L., Weinert L.A., Conway-Morris A., Cartwright R.A., Simpson A.J., Rambaut A., Nubel U., Fitzgerald J.R.: Recent human-to-poultry host jump, adaptation, and pandemic spread of Staphylococcus aureus. Proc Natl Acad Sci USA 2009, 106, 19545-19550.

19. Malachowa N., DeLeo F.R.: Mobile genetic elements of Staphylococcus aureus. Cell Mol Life Sci 2010, 67, 3057-3071.

20. Mehrotra M., Wang G., Johnson W.M.: Multiplex PCR for detection of genes for Staphylococcus aureus enterotoxins, exfoliative toxins, toxic shock syndrome toxin 1, and methicillin resistance. J Clin Microbiol 2000, 38, 1032-1035.

21. Momoh A.H., Kwaga J.K.P., Bello M., Sackey A.K.B., Larsen A.R.: Antibiotic resistance and molecular characteristics of Staphylococcus aureus isolated from backyard-raised pigs and pig workers. Trop Anim Health Prod 2018, 50, 1565-1571.

22. Moon J.S., Lee A.R., Jaw S.H., Kang H.M., Joo Y.S., Park Y.H., Kim M.N., Koo H.C.: Comparison of antibiogram, staphylococcal enterotoxin productivity, and coagulase genotypes among Staphylococcus aureus isolated from animal and vegetable sources in Korea. J Food Prot 2007, 70, 2541-2548.

23. Neopane P., Nepal H.P., Shrestha R., Uehara O., Abiko Y.: In vitro biofilm formation by Staphylococcus aureus isolated from wounds of hospital-admitted patients and their association with antimicrobial resistance. Int J Gen Med 2018, 11, 25-32.

24. Pinchuk I.V., Beswick E.J., Reyes V.E.: Staphylococcal enterotoxins. Toxins (Basel) 2010, 2, 2177-2197. 
25. Singh A.K., Prakash P., Achra A., Singh G.P., Das A., Singh R.K.: Standardization and Classification of In vitro Biofilm Formation by Clinical Isolates of Staphylococcus aureus. J Glob Infect Dis 2017, 9, 93-101.

26. Sommanustweechai A., Chanvatik S., Sermsinsiri V., Sivilaikul S., Patcharanarumol W., Yeung S., Tangcharoensathien V.: Antibiotic distribution channels in Thailand: results of key-informant interviews, reviews of drug regulations and database searches. Bull World Health Organ 2018, 96, 101-109.

27. Tam K., Torres V.J.: Staphylococcus aureus Secreted Toxins and Extracellular Enzymes. Microbiol Spectr 2019, 7, doi: 10.1128/microbiolspec.GPP3-0039-2018.

28. Wongboot W., Chomvarin C., Engchanil C., Chaimanee P.: Multiplex PCR for detection of superantigenic toxin genes in methicillin-sensitive and methicillin-resistant Staphylococcus aureus isolated from patients and carriers of a hospital in northeast Thailand. Southeast Asian J Trop Med Public Health 2013, 44, 660-671.

29. Wongboot W., Chomvarin C., Namwat W.: Phenotypic and Genotypic Detection of Enterotoxins, Toxic Shock Syndrome Toxin-1 and of Methicillin Resistance in Staphylococcus aureus Isolated from Retail Ready-to-Eat Foods in Northeastern Thailand. Southeast Asian J Trop Med Public Health 2015, 46, 97-104.

30. Xu Z., Liang Y., Lin S., Chen D., Li B., Li L., Deng Y.: Crystal Violet and XTT Assays on Staphylococcus aureus Biofilm Quantification. Curr Microbiol 2016, 73, 474-482.

31. Zhang Y., Wang Y., Cai R., Shi L., Li C., Yan H.: Prevalence of Enterotoxin Genes in Staphylococcus aureus Isolates from Pork Production. Foodborne Pathog Dis 2018, 15, 437-443. 